Ciência Florestal, Santa Maria, v. 26, n. 4, p. 1179-1188, out.-dez., 2016

ISSN 0103-9954

\title{
DINÂMICA DA CHUVA DE SEMENTES EM REMANESCENTE DE FLORESTA ESTACIONAL SUBTROPICAL
}

\section{DYNAMICS OF SEED IN REMAINING OF SUBTROPICAL SEASONAL FOREST}

\author{
Marta Silvana Volpato Sccoti ${ }^{1}$ Maristela Machado Araujo ${ }^{2}$ Thaíse da Silva Tonetto ${ }^{3}$ \\ Solon Jonas Longhi ${ }^{4}$
}

\section{RESUMO}

O presente estudo teve por objetivo avaliar a dinâmica da chuva de sementes em dois agrupamentos florísticos formados em um remanescente de Floresta Estacional Subtropical. Os agrupamentos foram caracterizados pelo estágio sucessional da floresta, denominados de grupo I (Floresta Secundária em Estágio Médio - FSEM), com predomínio de espécies secundárias iniciais; e o grupo II (Floresta Secundária em Estágio Avançado - FSEA), com predomínio de espécies secundárias tardias. A chuva de sementes (CS) foi avaliada em 70 coletores de $1 \mathrm{~m}^{2}$, distribuídos de forma aleatória nos dois agrupamentos, sendo que no grupo I foram instalados 15 coletores e no grupo II, por apresentar maior área, 55 coletores. O material depositado foi coletado mensalmente, no período de outubro de 2008 a outubro de 2010. A dinâmica da CS foi avaliada pela densidade de sementes de cada espécie, síndrome de dispersão e sazonalidade da produção. Utilizou-se o teste não paramétrico da Soma das Ordens de Wilcoxon (W) para verificar diferenças estatísticas nas médias de densidade de sementes em cada ano de avaliação para cada trecho de floresta. A chuva de sementes do remanescente estudado, durante os três anos de estudo, foi representada por 114 espécies e a maior produção de sementes ocorreu em 2008 (1632 sem.m $\mathrm{m}^{-2}$ no grupo I e $1270 \mathrm{sem} . \mathrm{m}^{-2}$ no grupo II), principalmente devido à ocorrência da alta produção de Chusquea ramosissima Lindm., Dasyphyllum spinescens (Less.) Cabrera, Vernonanthura discolor (Spreng.) H.Rob. Somente no segundo ano (2009) observaram-se diferenças estatísticas nas médias de densidade entre os dois grupos analisados $\left(\mathrm{FSEM}=565,6 \mathrm{sem} \cdot \mathrm{m}^{-2}\right.$ e FSEA $=274 \mathrm{sem} \cdot \mathrm{m}^{-2} ; \mathrm{Z}$ tabelado $=1,96$ e W FSEM x FSEA = 2,248). As espécies zoocóricas predominaram na área de estudo, e os períodos de maior produção de sementes foram o inverno e a primavera. A CS mostrou-se importante mecanismo de regeneração natural para o remanescente de floresta, sendo afetado pelas condições climáticas e estágio sucessional da floresta.

Palavras-chave: sucessão florestal; síndrome de dispersão; sazonalidade de produção.

\begin{abstract}
The aim of this study was to evaluate the dynamics of the seed rain in two Floristic groups formed in a remnant of Subtropical Seasonal Forest. The clusters were characterized by successional stage of the forest, namely group I (Secondary Forest in Intermediate Stage-FSEM), with a predominance of early secondary species, and group II (Secondary Forest in Advanced Stage-FSEA), with a predominance of late

1 Engenheira Florestal, $\mathrm{Dr}^{\mathrm{a}}$., Professora Adjunto do Departamento de Engenharia Florestal, Campus de Rolim de Moura, Universidade Federal de Rondônia, Av. Norte Sul, Bairro Nova Morada, 7300, CEP 7694000, Rolim de Moura (RO), Brasil.martasccoti@yahoo.com.br

2 Engenheira Florestal, Dr ${ }^{\mathrm{a}}$., Professora Adjunto do Departamento de Ciências Florestais, Centro de Ciências Rurais, Universidade Federal de Santa Maria, Av. Roraima, 1000, CEP 97105-900, Santa Maria (RS), Brasil. araujo.maristela@gmail.com

3 Engenheira Florestal, Doutoranda do Programa de Pós-Graduação em Engenharia Florestal, Centro de Ciências Rurais, Universidade Federal de Santa Maria, Av. Roraima, 1000, CEP 97105-900, Santa Maria (RS), Brasil. thaisetonetto@hotmail.com

4 Engenheiro Florestal, Dr., Professor Visitante do Departamento de Ciências Florestais, Centro de Ciências Rurais, Universidade Federal de Santa Maria, Av. Roraima, 1000, CEP 97105-900, Santa Maria (RS), Brasil. longhi.solon@gmail.com
\end{abstract}

Recebido para publicação em 25/04/2014 e aceito em 4/12/2014

Ci. Fl., v. 26, n. 4, out.-dez., 2016 
secondary species. Seed rain was evaluated in 70 collectors of $1 \mathrm{~m}^{2}$, randomly distributed in the two groups, whereas in group I were installed 15 collectors and in group II, due to its greater area, 55 collectors. The deposited material was collected monthly from October 2008 to October 2010. The dynamics of seed rain was assessed by the density of seeds of each species of dispersal and seasonal production. We used the nonparametric Test of Wilcoxon Sum of Orders (W) to determine statistical differences in the mean density of seeds in each year of assessment for each patch of forest. Seed rain in the remaining studied during three years of study, was represented by 114 species and higher seed production occurred in 2008 (1632-seeds. $\mathrm{m}^{-2}$ in group I and 1270-seeds. $\mathrm{m}^{-2}$ in group II), mainly due to the occurrence of high production Chusquea ramosissima Lindm., Dasyphyllum spinescens (Less.) Cabrera, Vernonanthura discolor (Spreng.) H.Rob. Only in the second year (2009) observed statistical differences in mean density between the two groups $\left(F S E M=565.6\right.$ seeds. $\mathrm{m}^{-2}$ and $\mathrm{FSEA}=274$ seeds. $\mathrm{m}^{-2} ; \mathrm{Z}=1.96$ and tabulated; W FSEM $\times$ FSEA =2.248). Zoochorous species predominated in the study area, and periods of increased seed production were the winter and spring. The seed rain proved important mechanism for natural regeneration of remnant forest and is affected by climate conditions and successional stage of the forest.

Keywords: forest succession; dispersion syndrome; seasonality of production.

\section{INTRODUÇÃO}

Os remanescentes florestais atualmente apresentam-se em diferentes níveis de alteração, formando "manchas" com diferentes características florísticas (LONGHI et al., 2006; GOMES et al., 2008) que, quando estudadas, permitem inferir sobre espécies potenciais para restabelecimentos de áreas, nas suas respectivas regiões. Esses ambientes complexos demonstram variações na sua estrutura arbórea, arbustiva, herbácea e de outras formas de vida que, consequentemente, influenciam os mecanismos de regeneração, como por exemplo, a chuva de sementes.

A chuva de sementes é representada pela dispersão de diásporos, sendo avaliada pela quantidade de sementes depositada em área específica por determinado tempo. Conforme Puig (2008), esse mecanismo é de grande importância para dinâmica da floresta, tanto na sucessão em clareiras como no fenômeno de substituição de plantas.

Assim, as sementes dispersadas na floresta podem ser provenientes do próprio local, promovendo a autorregeneração da floresta, ou trazidas de outros locais, o que significa o avanço da regeneração de indivíduos e espécies externas à área (HARPER, 1977; MARTINEZ-RAMOS; SOTOCASTRO, 1993), sendo que as sementes vindas de outros locais têm papel importante no processo sucessional, aumentando a riqueza florística local.

Nesse sentido, a dispersão de sementes na floresta pode ser feita por animais (zoocórica), pelo vento (anemocórica), pela água (hidrocórica), pela gravidade (barocórica) e pela própria planta (autocórica) (SIMPSON et al., 1989), assim, o conhecimento da síndrome de dispersão predominante em uma comunidade florestal permite inferir sobre a estrutura da vegetação, estágio sucessional e grau de conservação (PIVELLO et al., 2006). Dessa forma, em florestas tropicais, a maioria das espécies secundárias tardias de estratos intermediários apresenta dispersão por animais, enquanto as espécies secundárias iniciais dispersam pelo vento (HARPER, 1977).

Outro fator relevante na dispersão referese à variação sazonal na produção de sementes, que influencia o recrutamento das espécies vegetais, representando uma importante estratégia de regeneração da área. Desse modo, estudos que relacionam a eficiência da dispersão de espécies de florestas tropicais, o comportamento da dispersão em ambientes em diferentes estágios sucessionais são pouco abordados, podendo-se citar alguns exemplos encontrados em Vieira (1998) e Pivello et al. (2006).

Sendo assim, este trabalho teve por objetivo avaliar a dinâmica da chuva de sementes em dois agrupamentos de vegetação em diferentes estágios sucessionais em remanescente de Floresta Estacional Subtropical.

\section{MATERIAL E MÉTODOS}

\section{Localização e caracterização da área de estudo}

A área de estudo pertence ao Ministério da Defesa, apresenta cerca de 560 ha e está localizada 
no Campo de Instrução de Santa Maria (CISM), no município de Santa Maria - RS (5352'O e $29^{\circ} 46^{\prime}$ 'S).

As principais classes de solo da região pertencem à Unidade de Mapeamento Santa Maria, denominado Argissolo Bruno-Acinzentado Alítico Úmbrico e Argissolo Amarelo Alítico Típico, originados de siltitos e arenito, que ocorrem em duas situações de paisagem: uma dominando o relevo suavemente ondulado e outra ocupando áreas de coxilhas em cotas intermediárias entre Argissolos Vermelhos (Unidade São Pedro) (STRECK et al., 2008).

De acordo com a classificação climática de Köppen, a região da área de estudo caracterizase pelo clima do tipo $\mathrm{Cfa}$, com temperatura média de 17,9 a $19,2^{\circ} \mathrm{C}$. A precipitação média anual está entre 1400 e $1760 \mathrm{~mm}$, podendo ocorrer chuvas torrenciais de $182 \mathrm{~mm}$, em 24 horas, geadas de abril a novembro e períodos secos de novembro a janeiro (LEMOS et al., 1973).

A região fitogeográfica da área de estudo pertence à Floresta Estacional Decidual, também denominada de Floresta Estacional Subtropical por Schumacher et al. (2011), sendo considerada uma vegetação quase que exclusiva das bacias dos rios Ibicuí, Jacuí, Santa Maria e Uruguai (VELOSO et al., 1991). Esta formação encontra-se bastante descaracterizada de sua composição original, devido à exploração no passado dos seus principais elementos florísticos.

Estudos realizados nesta tipologia florestal apresentam a predominância das famílias Myrtaceae, Lauraceae, Euphorbiaceae, Fabaceae, Sapindaceae (FARIAS et al., 1994; VACCARO et al., 1999; LONGHI et al., 2000; ARAUJO et al., 2004) e segundo Leite e Klein (1990), observa-se a formação de três estratos bem definidos, o estrato superior, composto por espécies como a Apuleia leiocarpa (Vogel) J.F.Macbr., Cordia trichotoma (Vell.) Arrabida ex Steudel., Parapiptadenia rigida (Benth.) Brenan, Cedrela fissilis Vell., Holocalyx balansae Micheli, Peltophorum dubium (Spreng.) Taub. e Enterolobium Contortisiliquum (Vell.) Morong; o segundo estrato que constitui a parte mais densa do interior da floresta, sendo formado basicamente por espécies da família das Lauraceas e das leguminosas dos gêneros Lonchocarpus, Parapiptadenia e Apuleia; e o terceiro estrato o das arvoretas, representado pelas espécies Actinostemon concolor (Spreng.) Müll.Arg. e Sorocea bonplandii (Baill.) W.C. Burger, Lanjouw $\&$ Boer, principalmente.
Sccoti (2012) estudando a vegetação com circunferência a altura do peito (CAP) $\geq 30 \mathrm{~cm}$ identificou a formação de dois grupos florísticos, caracterizados por apresentarem-se em diferentes estágios de sucessão (Figura 1).

O grupo I representou a Floresta Secundária em Estágio Médio (FSEM) de sucessão, observada em área de mais fácil acesso, na qual ainda existem indicativos de uma antiga estrada, o que sugere maior influência de extração de madeira nesse ponto.

Já o grupo II, denominado de Floresta Secundária em Estágio Avançado (FSEA) de sucessão, caracterizou-se por ocorrer em local com a presença de canais de drenagem, que, associados à fragilidade do solo, favoreceram a formação de voçorocas, dificultando o acesso a essa área, tornando, assim, inviável a exploração, o que, possivelmente, favoreceu a conservação do ambiente.

Conforme Sccoti (2012), o trecho de floresta, considerado como Floresta Secundária em Estágio Médio (FSEM) de sucessão, apresentou como espécies indicadoras: Casearia sylvestris Sw. e Sebastiania commersoniana (Baill.) L.B. Sm. \& Downs e espécies preferenciais, Casearia sylvestris, Cordia americana (L.) Gottshling \& J.E.Mill.,

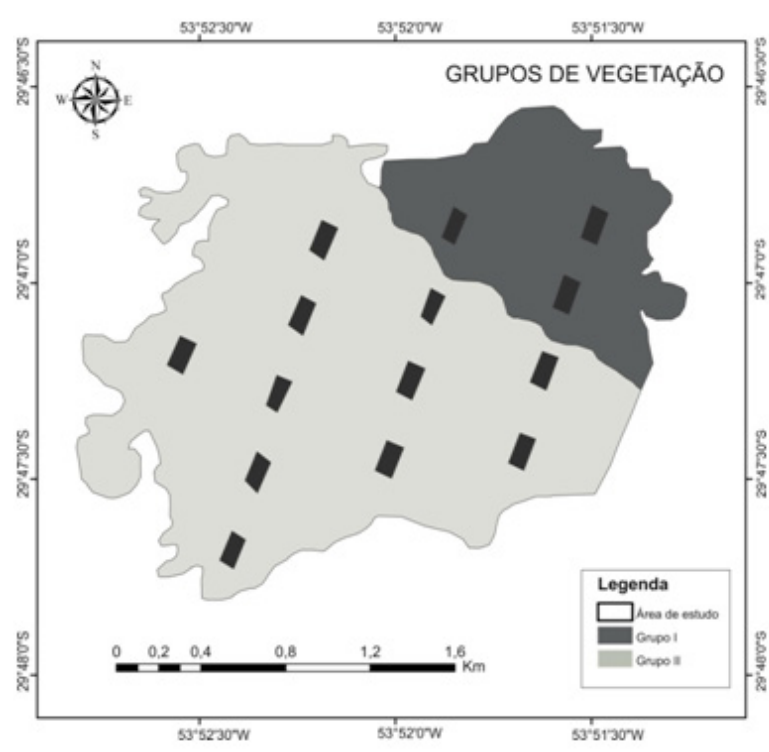

FIGURA 1: Grupos florísticos observados em remanescente de Floresta Estacional Subtropical, Santa Maria - RS.

FIGURE 1: Floristic groups observed in remaining Subtropical Seasonal Forest, Santa Maria, RS state. 
Luehea divaricata Mart., Parapiptadenia rigida e Sebastiania commersoniana. Essas espécies são caracterizadas como secundárias iniciais, o que indica sua adaptação em ambientes com maior disponibilidade de luz e, ainda, Casearia sylvestris e Sebastiania commersoniana são caracterizadas por ocorrerem em ambientes com maior presença de umidade.

$\mathrm{Na}$ Floresta Secundária em Estágio Avançado (FSEA) de sucessão, as espécies indicadoras foram Trichilia claussenii C.DC., Plinia rivularis (Cambess.) e Cupania vernalis Cambess., e as espécies preferenciais foram Chrysophylum marginatum (Hook. \& Arn.) Radlk., Cupania vernalis, Myrcianthes pungens (Berg) Legr, Nectandra megapotamica (Spreng.) Mez., Plinia rivularis e Trichilia claussenii. Essas espécies são caracterizadas como esciófilas, ou adaptadas à luz difusa, higrófilas e abundantes no estrato médio das matas, no estado do Rio Grande do Sul.

\section{Metodologia}

No ano de 2007, foram marcados, na área de estudo, 14 blocos de $20 \times 100$ m distribuídos de forma sistemática, em quatro faixas equidistantes $500 \mathrm{~m}$, com comprimento variável em função dos limites da floresta. Os blocos de $20 \times 100 \mathrm{~m}$ foram divididos em parcelas de 10 x $10 \mathrm{~m}$. Para instalação dos coletores de chuva de sementes foram sorteadas cinco parcelas em cada bloco, totalizando na área 70 unidades amostrais, sendo que, dessas, 15 representaram o grupo I - FSEM e 55 no grupo II - FSEA

Os coletores possuíam área de $1 \mathrm{~m}^{2} \mathrm{e}$ foram confeccionados em tela de polietileno $\left(\right.$ sombrite $^{\odot}$ ) sob cano de PVC instalados a $50 \mathrm{~cm}$ do solo. O monitoramento da chuva de sementes foi realizado durante o período de outubro de 2007 a outubro de 2010, com coletas realizadas mensalmente.

\section{Análise dos dados}

Em cada grupo florístico identificado na vegetação analisou-se a composição florística, índice de diversidade de Shanonn, índice de equitabilidade de Pielou e densidade de propágulos por estações do ano (verão: janeiro, fevereiro e março; outono: abril, maio e junho; inverno: julho, agosto e setembro; primavera: outubro, novembro e dezembro).

Os dados de densidade média de indivíduos em cada ano de avaliação para cada grupo florístico
(FSEM e FSEA) foram analisados pelo teste não paramétrico da Soma das Ordens de Wilcoxon (W) (CARNELUTTI FILHO et al., 2001), que permite verificar se existe superioridade de um grupo em relação ao outro, em um conjunto de dados que não apresentam distribuição normal.

A identificação dos frutos e sementes e a sua síndrome de dispersão foi efetuada por comparação com mostruário (Bolsa de Sementes do Laboratório de Silvicultura e Viveiro Florestal - Departamento de Ciências Florestais - UFSM), bibliográficas específicas (LORENZI, 2002a; LORENZI, 2002b; BACKES; IRGANG, 2002; CARVALHO, 2003; 2006), assim como, por consultas a especialistas.

Quando não foi possível a identificação da síndrome de dispersão das sementes pela bibliografia, realizou-se uma análise do tamanho, cor e morfologia dos propágulos, classificando-as de acordo com Van Der Pijl (1982), como anemocóricas (dispersão pelo vento), quando apresentavam estruturas ou alas que permitissem planar; e como zoocóricas, aquelas que apresentavam características de dispersão por animais, em especial frutos drupáceos, bagas e sementes ariladas.

\section{RESULTADOS E DISCUSSÃO}

A chuva de sementes do remanescente estudado, durante três anos de estudo, foi representada por 114 espécies (Tabela 1). Destas, 47\% (54 morfoespécies) não foram identificadas, quatro foram identificadas em nível de família e quatro em nível de gênero.

Outros estudos também mostram a dificuldade de identificação dos propágulos provenientes da chuva de sementes. Chami et al. (2011) observaram, em Floresta Ombrófila Mista, 55 espécies, em estudo de chuva de sementes, sendo que em $44 \%$ das espécies amostradas não foi possível a identificação. De forma semelhante, Fernandes (2006) identificou 96 espécies somente como morfoespécies, em estudo de chuva de sementes, em áreas com diferentes estágios sucessionais, na Floresta Ombrófila Densa Montana, em Miguel Pereira - RJ.

As famílias que apresentaram maior riqueza de espécies na chuva de sementes foram Myrtaceae, Asteraceae, Meliaceae e Sapindaceae, as quais também foram descritas como predominantes em outras pesquisas abordando a chuva de sementes (ARAUJO et al. 2004; SCHERER; JARENKOW, 2006; BUDKE et al., 2005). 
TABELA 1: Riqueza de espécies, síndrome de dispersão (SD) e densidade Absoluta (DA) de sementes observadas durante três anos em estudo de chuva de sementes em dois grupos florísticos de Floresta Estacional Subtropical, Santa Maria, RS.

TABLE 1: Species richness, dispersal syndrome (SD) and Absolute density (DA) of seeds observed during three years in a study of seed rain in two groups floristic of Subtropical Forest, Santa Maria, RS state.

\begin{tabular}{|c|c|c|c|c|}
\hline \multirow{3}{*}{ Família } & \multirow{3}{*}{ Espécie } & \multirow{3}{*}{ SD } & FSEM & FSEA \\
\hline & & & $\mathrm{DA}\left(\mathrm{sem} . \mathrm{m}^{2}\right)$ & $\mathrm{DA}\left(\mathrm{sem} \cdot \mathrm{m}^{2}\right)$ \\
\hline & & & $2008 / 2009 / 2010$ & $2008 / 2009 / 2010$ \\
\hline Annonaceae & Annona neosalicifolia H.Rainer & Zoo & $0,07 / 0,07 / 0$ & $0,17 / 0,16 / 0,11$ \\
\hline Araliaceae & $\begin{array}{l}\text { Schefflera morototoni (Aubl.) Maguire et } \\
\text { al. }\end{array}$ & Ane & $0 / 0,07 / 0$ & $0,6 / 0,15 / 1,16$ \\
\hline \multirow{5}{*}{ Asteraceae } & NI & Ane & $0 / 0,07 / 0$ & $0,13 / 0 / 0$ \\
\hline & Chaptalia nutans (L.) Polack & Ane & $0,07 / 0 / 0$ & $0,02 / 0 / 0$ \\
\hline & Dasyphyllum spinescens (Less.) Cabrera & Ane & $511 / 62,3 / 12,7$ & $172,9 / 24,53 / 5,5$ \\
\hline & Syagrus romanzoffiana (Cham.) Glassman & Zoo & $53,5 / 57,9 / 48,2$ & $1,4 / 3,7 / 8,75$ \\
\hline & Vernonanthura discolor (Spreng.) H.Rob. & Ane & $69,5 / 196,7 / 63$ & 174,3 / 60,35 / 39,4 \\
\hline \multirow{3}{*}{ Bignoniaceae } & NI & Ane & $4,3 / 6,5 / 9,4$ & $13,15 / 2,45 / 0,16$ \\
\hline & $\begin{array}{l}\text { Dolichandra unguis-cati (L.) } \\
\text { L.G.Lohmann }\end{array}$ & Ane & $1,13 / 0 / 0$ & $0,23 / 0 / 0$ \\
\hline & Handroanthus heptaphyllus Mattos & Ane & $1,8 / 0,07 / 0,27$ & $0 / 0 / 0,24$ \\
\hline \multirow{2}{*}{ Boraginaceae } & $\begin{array}{l}\text { Cordia americana (L.) Gottshling \& } \\
\text { J.E.Mill. }\end{array}$ & Ane & $23,4 / 20,8 / 193$ & $19,1 / 34,65 / 105,24$ \\
\hline & Cordia trichotoma (Vell.) Arrab. ex Steud. & Ane & $0 / 12,7 / 0$ & 8,42 / 27,87 / 21,67 \\
\hline Cannabaceae & Celtis iguanea (Jacq.) Sarg. & Zoo & $1 / 0 / 0,53$ & $0 / 0 / 0,11$ \\
\hline Combretaceae & Combretum leprosum Mart. et Eicher & Ane & $0,2 / 2,27 / 11,73$ & $0 / 4,2 / 6,4$ \\
\hline Cyperaceae & NI & Ane & $0 / 0,67 / 0$ & $0 / 0,20 / 0$ \\
\hline Erythroxylaceae & Erythroxylum deciduum A.St.-Hil. & Zoo & $0,47 / 0,07 / 5,47$ & $0,15 / 0 / 0,26$ \\
\hline \multirow[b]{2}{*}{ Euphorbiaceae } & Actinostemon concolor Spreng. & Zoo & $70,13 / 11,6 / 12,33$ & $131,53 / 19,91 / 22,56$ \\
\hline & $\begin{array}{l}\text { Sebastiania commersoniana (Baill.) L. B. } \\
\text { Sm. et Downs }\end{array}$ & Aut & $8,53 / 2,93 / 13,4$ & $7,62 / 0,2 / 6,95$ \\
\hline \multirow{4}{*}{ Fabaceae } & Parapiptadenia rigida (Benth.) Brenan & Ane & $1,47 / 25 / 0$ & $1,42 / 7 / 0,35$ \\
\hline & $\begin{array}{l}\text { Senegalia bonariensis (Gillies ex Hook. \& } \\
\text { Arn.) Seigler \& Ebinger }\end{array}$ & Ane & $0 / 0,07 / 0,27$ & $0 / 1,75 / 0,04$ \\
\hline & Mimosa bimucronata (DC.) Kuntze & Aut & $0 / 0 / 0$ & $0,55 / 0 / 0$ \\
\hline & $\begin{array}{l}\text { Enterolobium contortisiliquum (Vell.) } \\
\text { Morong }\end{array}$ & Zoo & $0 / 0,07 / 0$ & $0,13 / 0,56 / 0,02$ \\
\hline Hipocrateaceae & Anthodon sp. & Ane & $24,8 / 3,87 / 2,67$ & $2,04 / 2,51 / 1,62$ \\
\hline \multirow{2}{*}{ Lauraceae } & Aiouea saligna Meisn. & Zoo & $0 / 0,07 / 0,27$ & $0,08 / 0 / 0,02$ \\
\hline & Nectandra megapotamica (Spreng.) Mez. & Zoo & $0,4 / 0,4 / 0$ & $0,04 / 1,15 / 0,02$ \\
\hline Loranthaceae & $\begin{array}{l}\text { Struthanthus flexicaulis (Mart. ex Schult. } \\
\text { f.) Mart. }\end{array}$ & Ane & $2,1 / 2,2 / 0,53$ & $3,19 / 0,42 / 0,64$ \\
\hline Malvaceae & Luehea divaricata Mart. \& Zucc. & Ane & $4,27 / 2,67 / 5,53$ & $0,26 / 0,64 / 1,9$ \\
\hline
\end{tabular}


TABELA 1: Continuação...

TABLE 1: Continued...

\begin{tabular}{|c|c|c|c|c|}
\hline \multirow{3}{*}{ Família } & \multirow{3}{*}{ Espécie } & \multirow{3}{*}{ SD } & FSEM & FSEA \\
\hline & & & $\mathrm{DA}\left(\mathrm{sem} \cdot \mathrm{m}^{2}\right)$ & $\mathrm{DA}\left(\mathrm{sem} \cdot \mathrm{m}^{2}\right)$ \\
\hline & & & $2008 / 2009 / 2010$ & $2008 / 2009 / 2010$ \\
\hline \multirow{4}{*}{ Meliaceae } & Cedrela fissilis Vell. & Ane & $0,07 / 1 / 0,07$ & $0,34 / 2,42 / 0,15$ \\
\hline & Trichilia catigua A. Juss & Zoo & $0 / 0 / 0$ & $0 / 0 / 0,06$ \\
\hline & Trichilia claussenii C.DC. & Zoo & $0 / 1,33 / 0$ & $0,26 / 12,1 / 0,13$ \\
\hline & Trichila elegans A. Juss. & Zoo & $0 / 1,2 / 0,53$ & $0,02 / 2,16 / 0,1$ \\
\hline Menispermaceae & Abuta sp. & Zoo & $0 / 0 / 0$ & $0,08 / 0 / 0,02$ \\
\hline Moraceae & Ficus luschnathiana (Miq.) Miq. & Zoo & $0,07 / 0 / 0$ & $2,21 / 2,16 / 8,71$ \\
\hline \multirow{2}{*}{ Myrsinaceae } & Myrsine coriacea (Sw.) R.Br. & Zoo & $0 / 0,33 / 0,07$ & $0 / 0,18 / 0,06$ \\
\hline & Myrsine sp. & Zoo & $0 / 0 / 0,13$ & $0 / 0,02 / 0$ \\
\hline \multirow{7}{*}{ Myrtaceae } & Campomanesia xanthocarpa O. Berg & Zoo & $0,33 / 0 / 0$ & $0,17 / 2,4 / 0$ \\
\hline & Eugenia involucrata DC. & Zoo & $0,13 / 0 / 0$ & $3,13 / 0,84 / 0,76$ \\
\hline & Eugenia rostrifolia Legr. & Zoo & $0,2 / 0 / 0$ & $0,02 / 0 / 0$ \\
\hline & Eugenia uniflora Linnaeus & Zoo & $0,53 / 2,93 / 0,13$ & $0,04 / 1,49 / 1,06$ \\
\hline & Myrcianthes pungens (O. Berg) D. legrand & Zoo & $0 / 1,2 / 0,4$ & $0 / 5,8 / 0$ \\
\hline & NI & Zoo & $0 / 0,07 / 0,8$ & $0 / 0,02 / 0$ \\
\hline & Plinia rivularis (Camb.) Rotman & Zoo & $5,33 / 0 / 0$ & $0,19 / 0,25 / 0,06$ \\
\hline \multirow{2}{*}{ Phytolaccaceae } & Phytolacca dioica $\mathrm{L}$. & Zoo & $0,27 / 0 / 0,27$ & $9,7 / 0,45 / 5,3$ \\
\hline & Seguieria aculeata Jacq. & Ane & $2 / 9,13 / 0,07$ & 0,55 / 6,58 / 0,02 \\
\hline Poaceae & Chusquea ramosissima Lindm. & Aut & $801,4 / 0$ / 0 & $588,9 / 0 / 0$ \\
\hline Polygonaceae & Ruprechtia laxiflora Meisn. & Ane & $1,6 / 92,47 / 459,2$ & $6,09 / 2,27 / 61,87$ \\
\hline \multirow{2}{*}{ Rosaceae } & Eriobotrya japonica (Thunb.) Lindl. & Zoo & $0 / 0 / 0$ & $0 / 0,02 / 0$ \\
\hline & Prunus myrtifolia L. Urb. & Zoo & $0 / 0,87 / 0$ & $0,04 / 0,35 / 0,15$ \\
\hline \multirow{3}{*}{ Rutaceae } & Citrus sinensis (L.) Osbeck & Zoo & $0,47 / 1 / 0$ & $0 / 0,1 / 0$ \\
\hline & Helietta apiculata Benth. & Ane & $8,93 / 5,47 / 8,53$ & $37,25 / 3,2 / 22,66$ \\
\hline & Pilocarpus pennatifolius Lem. & Zoo & $0 / 0 / 0$ & $1,13 / 0,27 / 0,78$ \\
\hline Salicaceae & Banara tomentosa Clos & Zoo & $0 / 0 / 0$ & $0 / 0,08 / 0$ \\
\hline \multirow{4}{*}{ Sapindaceae } & $\begin{array}{l}\text { Allophylus edulis (A.St.-Hil., Cambess. \& } \\
\text { A. Juss.) Radlk. }\end{array}$ & Zoo & $0,27 / 0,07 / 0,07$ & $0,49 / 1,11 / 1,2$ \\
\hline & Cupania vernalis Camb. & Zoo & $4,87 / 2,33 / 3,47$ & $7,3 / 4,31 / 5,4$ \\
\hline & Matayba elaeagnoides Radlk. & Zoo & $0 / 0 / 0,2$ & $2,77 / 0,55 / 10,33$ \\
\hline & Urvillea sp. & Ane & $20,8 / 4,13 / 8,53$ & $51,4 / 1,95 / 61$ \\
\hline \multirow{2}{*}{ Sapotaceae } & $\begin{array}{l}\text { Chrysophyllum gonocarpum (Mart. \& } \\
\text { Eichler) Engl. }\end{array}$ & Zoo & $0 / 0 / 0$ & $0,77 / 0,2$ / 0 \\
\hline & $\begin{array}{l}\text { Chrysophyllum marginatum (Hook. \& } \\
\text { Arn.) Radlk. }\end{array}$ & Zoo & $0,33 / 0,13 / 0$ & $4,83 / 1,07 / 0,07$ \\
\hline Violacecea & Anchietea pyrifolia (Mart.) G.Don. & Ane & $0,07 / 0 / 0$ & $0,08 / 0,02 / 0,02$ \\
\hline NI & - & - & $6,73 / 32,87 / 2,67$ & $14,13 / 30,24 / 15,52$ \\
\hline
\end{tabular}

Em que: FSEM = Floresta Secundária em Estágio Médio de Sucessão; FSEA = Floresta Secundária em Estágio Avançado de Sucessão; Ane = Anemocórica; Zoo: Zoocórica; Aut = Autocórica; NI = espécies não identificadas . 
$\mathrm{Na}$ área de estudo, as famílias Myrtaceae, Meliaceae e Sapindaceae também representam maior riqueza de espécies na vegetação adulta $(\mathrm{CAP} \geq 30 \mathrm{~cm})$, sub-bosque e banco de plântulas (SCCOTI, 2012), demonstrando que a chuva de sementes é um importante mecanismo para a manutenção dessas famílias na área.

Os agrupamentos florísticos, em termos de densidade de sementes, apresentaram diferenças estatísticas somente no segundo ano de estudo (2009) $\left(\mathrm{FSEM}=565,6 \quad \mathrm{sem} \cdot \mathrm{m}^{-2}\right.$ e $\mathrm{FSEA}=274$ sem. $\left.\mathrm{m}^{-2}\right)$. A diferença foi indicada pelos valores de $W$ calculado $>Z$ tabelado $(p<0,05) \quad(Z$ tabelado $=1,96)(\mathrm{W}$ FSEM x FSEA $=1,082-$ ANO 1; W FSEM x FSEA = 2,248 - ANO 2; W FSEM x FSEA $=1,32618-$ ANO 3 ).

A FSEA apresentou maior número de espécies na chuva de sementes, quando comparado à FSEM nos três anos de avaliação (Tabela 2). Esse resultado foi semelhante ao verificado por Vieira (1998) que observou redução gradativa do número de espécies do ecossistema mais conservado para os de maior alteração. Esse comportamento está associado à maior riqueza de espécies em ambientes mais conservados, o que favorece também a maior riqueza na chuva de sementes.

Os Índices de Shannon e Equitabilidade de Pielou foram baixos nos dois trechos de floresta. Porém, isso é considerado comum em estudos de chuva de sementes, uma vez que se observa o predomínio de poucas espécies com elevada abundância de sementes, confirmando o que ocorreu, por exemplo, no primeiro ano, quando apenas duas espécies foram responsáveis por $80 \%$ do total de sementes dispersadas na FSEM, sendo Chusquea ramosissima (801 sem.m $\left.{ }^{-2}\right)$ e Dasyphyllum spinescens $\left(511 \mathrm{sem} \cdot \mathrm{m}^{-2}\right)$. E, na FSEA, quatro espécies foram responsáveis por $83 \%$ da produção total nesse período, sendo elas Chusquea ramosissima $\left(588,89\right.$ sem. $\left.\mathrm{m}^{-2}\right)$, Vernonanthura discolor $\left(174,30 \mathrm{sem} . \mathrm{m}^{-2}\right)$, Dasyphyllum spinescens $\left(172,89\right.$ sem. $\left.\mathrm{m}^{-2}\right)$, Actinostemon concolor $(131,53$ sem. $\left.\mathrm{m}^{-2}\right)$. A mesma tendência também foi observada nos demais períodos de avaliação.

$\mathrm{O}$ trecho de FSEM apresentou maior produção média anual de sementes $\left(1.021 \mathrm{sem} . \mathrm{m}^{-2}\right.$. ano $^{-1}$ ), quando comparada com a do trecho de FSEA $\left(655 \mathrm{sem} . \mathrm{m}^{-2}\right.$.ano $\left.{ }^{-1}\right)$. O resultados são semelhantes aos obtidos por Vieira (1998), que verificou 883 sem. $\mathrm{m}^{-2}$ em floresta com 5 anos, 537 sem. $\mathrm{m}^{-2} \mathrm{em}$ floresta com 10 anos, 514 sem.m m $^{-2}$ em floresta de 20 anos e 220 sem. $\mathrm{m}^{-2}$ em floresta madura, na Amazônia.

A maior produção de sementes no trecho de FSEM está associada à alta produção de sementes de espécies pioneiras e secundárias iniciais (Chusquea ramosissima, Dasyphyllum spinescens, Vernonanthura discolor, Syagrus romanzoffiana, Cordia americana e Cordia trichotoma) (Tabela 1), que encontram, nesse local da floresta, ambientes com condições de maior luminosidade favorecendo a sua produção.

A maior produção de sementes ocorreu no primeiro ano para ambos os trechos de floresta analisados, diminuindo a produção no segundo ano e aumentando, novamente, no terceiro ano.

$\mathrm{O}$ primeiro ano apresentou maior produção, principalmente, por causa de Chusquea ramosissima. Somente essa espécie representou $49,1 \%$ da produção total quando comparada às demais espécies na FSEM e de $46,4 \%$ na FSEA. Chami et al. (2011), também observaram uma única espécie como responsável pela alta densidade em estudo de chuva de sementes em Floresta Ombrófila

TABELA 2: Dinâmica da chuva de sementes durante três anos de avaliação, em remanescente de Floresta Estacional Subtropical, Santa Maria - RS.

TABLE 2: Dynamics of seed rain for three years of evaluation, remaining Subtropical Seasonal Forest, Santa Maria, RS state.

\begin{tabular}{lcccccc}
\hline \multirow{2}{*}{ Características da População } & \multicolumn{3}{c}{ FSEM } & \multicolumn{3}{c}{ FSEA } \\
\cline { 2 - 7 } & 2008 & 2009 & 2010 & 2008 & 2009 & 2010 \\
\hline N. Espécies & 34 & 54 & 36 & 66 & 78 & 60 \\
Índice de Shanonn & 1,49 & 2,31 & 1,58 & 1,85 & 2,91 & 2,50 \\
Índice de Equitabilidade & 0,15 & 0,25 & 0,17 & 0,17 & 0,30 & 0,25 \\
Densidade total $\left(\right.$ sem.m $\left.^{-2}\right)$ & 1632 & 566 & 865 & 1270 & 274 & 420 \\
\hline
\end{tabular}

Em que: FSEM = Floresta Secundária em Estágio Médio; FSEA = Floresta Secundária em Estágio Avançado. 
Mista, no Rio Grande do Sul, neste caso, a espécie que se destacou foi Vernonanthura discolor.

No ano de 2009, no período de outubro até novembro, ocorreram fortes chuvas na região, o que pode ter comprometido a produção de sementes, nesse ano, já que chuvas fortes podem danificar as flores e também prejudicar a atividade dos polinizadores (JACKSON, 1978).

A síndrome de dispersão predominante nos dois agrupamentos foi a zoocórica. Sendo que o trecho de FSEA apresentou taxa um pouco mais elevada de espécies com essa síndrome quando comparada à FSEM (Figura 2).

O tipo de dispersão predominante pode servir como indicativo do estágio de sucessão da floresta, pois, segundo Fenner (1985), a alta quantidade de espécies zoocóricas é um indicativo de comunidades vegetais em estágios mais avançados de sucessão. Já, as espécies anemocóricas são mais comuns em clareiras, em que a dispersão pelo vento se torna mais eficientes do que por animais (PUIG, 2008). Assim, percebe-se que a FSEM está se restabelecendo por benefício do trecho de FSEA, o qual, possivelmente representa importante fonte

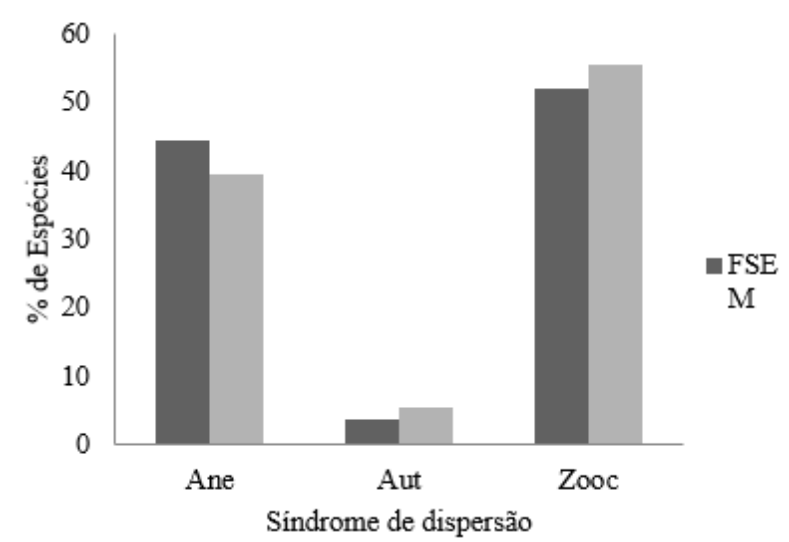

FIGURA 2: Síndromes de dispersão de sementes observada em dois trechos de Floresta Estacional Subtropical, Santa Maria - RS. Em que: FSEM = Floresta Secundária em Estágio Médio de sucessão e FSEA = Floresta Secundária em Estágio Avançado de sucessão.

FIGURE 2: Syndromes seed dispersal observed in two Subtropical Forest area, Santa Maria, RS state. Where: FSEM = Secondary Forest in Intermediate Stage and FSEA = Secondary Forest Succession in Advanced Stage. de sementes de espécies de estágio mais avançado.

Não foi observada uma tendência muito clara em relação à sazonalidade, pois o período de maior produção no primeiro e segundo ano de estudo foi verificado no inverno, com $61,1 \%$ e $39,4 \%$ da produção total, respectivamente, já no terceiro ano, a maior produção ocorreu na primavera com 48,9 $\%$ da produção total de sementes na área (Figura 3).

Vale ressaltar que, no primeiro ano, em 2008, a espécie Chusquea ramosissima, que frutificou no mês de agosto foi responsável pela maior taxa de produção de sementes. Essa espécie caracterizase por apresentar frutificação rara, podendo levar anos para promover uma nova frutificação. Assim, a sua frutificação em 2008, no período do inverno, pode ter alterando o comportamento da produção de sementes nesse período do ano.

Destaca-se ainda, que em 2009, as fortes chuvas que ocorreram na primavera (outubro a dezembro) podem ter prejudicado suas taxas de produção de sementes, favorecendo novamente a maior produção de sementes no inverno.

Variações nos períodos de produção de sementes de um ano para outro, também foram verificadas por Araujo et al. (2004), que analisaram, durante dois anos, a chuva de sementes na Floresta Estacional Decidual Ripária em Cachoeira do Sul - RS. Os autores observaram maior produção

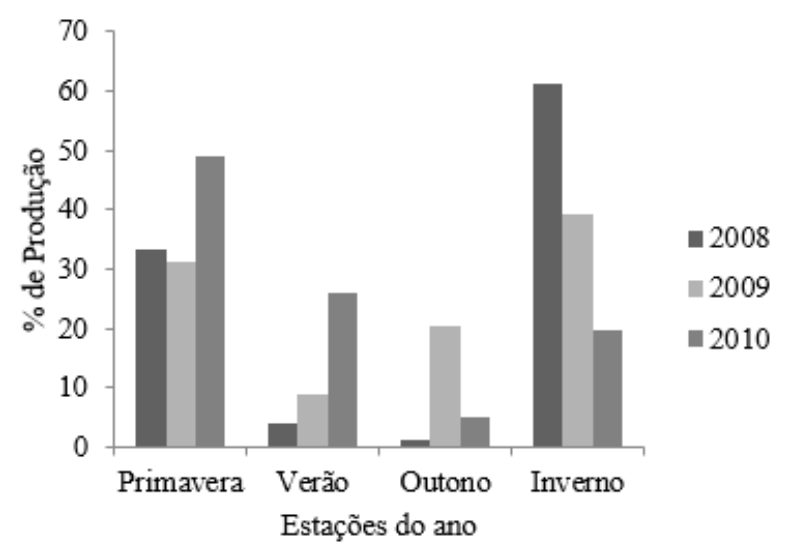

FIGURA 3: Produção de sementes observadas na chuva de sementes por estações do ano, em Remanescente de Floresta Estacional Subtropical, Santa Maria RS.

FIGURE 3: Production of seeds in the seed rain observed by seasons in Subtropical Remaining Seasonal Forest, Santa Maria, RS state. 
de sementes nos meses de setembro a janeiro no primeiro ano e no ano seguinte, observaram pico de produção em abril e maio, diminuindo a produção nos meses subsequentes e, aumentando novamente, de setembro a novembro.

$\mathrm{O}$ período que corresponde ao verão e outono tiveram as menores taxas de produção de sementes em todos os períodos de observação. Essa característica na sazonalidade de produção de sementes, nestes períodos do ano, está ligada principalmente às condições climáticas, pois, segundo Kageyama e Piña-Rodrigues (1993), a formação de gemas reprodutivas sofre influência das condições climáticas.

No verão, por exemplo, as baixas precipitações podem ser responsáveis por este fator, já no outono inicia-se o período de baixas temperaturas, o que ocasiona a perda de folhas da vegetação, provavelmente, influenciando de forma negativa no processo fotossintético das plantas e, assim, segundo Larcher (2000), a translocação de fotossintatos para o processo reprodutivo.

\section{CONCLUSÕES}

A chuva de sementes para o remanescente estudado apresentou importante componente no potencial de regeneração da floresta e das áreas adjacentes, apesar de apresentar alta densidade representada por poucas espécies.

Aproduçãode sementes, durantes os três anos de avaliação, demonstrou ser bastante influenciada pelas variações climáticas, indicando que, para melhor descrição dos fenômenos associados à dinâmica deste mecanismo de regeneração, se torna importante o estudo em períodos prolongados.

As espécies arbóreas demonstraram sazonalidade de produção que interfere também nos períodos de maior produção de sementes durante o ano.

O trecho de floresta em estágio mais avançado de sucessão, neste estudo, apresentou maior número de espécies contribuindo para a chuva de sementes, por outro lado, a densidade de sementes foi maior no trecho menos conservado, devido, principalmente, à alta produção de espécies heliófilas que predominam nesses ambientes.

O estudo da chuva de sementes pode ser uma ferramenta importante no diagnóstico do estágio sucessional da floresta e no grau de conservação das espécies.

\section{AGRADECIMENTOS}

Ao Programa de Pós-Graduação em Engenharia Florestal, da Universidade Federal de Santa Maria;

À $3^{\mathrm{a}}$ Divisão do Exército e à Direção do CISM pela disponibilidade da área para realização do estudo; e, à CAPES pela concessão de bolsa de estudo.

\section{REFERÊNCIAS BIBLIOGRÁFICAS}

ARAUJO, M. M. et al. Caracterização da chuva de sementes, banco de sementes do solo e banco de plântulas em Floresta Estacional Decidual Ripária Cachoeira do Sul, RS, Brasil. Scientia Forestalis, Piracicaba, n. 66, p. 128-141, dez. 2004.

BACKES, P.; IRGANG, B. Árvores do Sul: guia de identificação e reconhecimento ecológico. Porto Alegre: Pallotti, 2002. 325 p.

BUDKE, J. C. et al. Composição florística e estratégias de dispersão de espécies lenhosas em uma floresta ribeirinha, arroio Passo das Tropas, Santa Maria, RS, Brasil. Iheringia, Série Botânica, Porto Alegre, v. 60, n. 1, p. 17-24, jan./jun. 2005.

CARNELUTTI FILHO, A. C. et al. Testes não paramétricos para pesquisas agrícolas. Santa Maria: UFSM/CCR/Departamento de fitotecnia, 2001. 87 p.

CARVALHO, P. E. R. Espécies arbóreas brasileiras. Brasília: Embrapa Informações Tecnológicas; Colombo: Embrapa Florestas, 2003. v. $1,1039 \mathrm{p}$.

CARVALHO, P. E. R. Espécies arbóreas brasileiras. Brasília: Embrapa Informações Tecnológicas; Colombo, PR: Embrapa Florestas, 2006. v. 2, 627 p.

CHAMI, L. B. et al. Mecanismos de regeneração natural em diferentes ambientes de remanescente de Floresta Ombrófila Mista, São Francisco de Paula, RS. Ciência Rural, Santa Maria, v. 41, n. 2 , p. 251-259, fev. 2011.

FARIAS, J. A. C. et al. Estrutura fitossociológica de uma Floresta Estacional Decidual na região de Santa Maria, RS. Ciência Florestal, Santa Maria, v. 1, n. 4, p. 109-128, 1994.

FENNER, M. Seed ecology. London: Chapman and Hall, 1985. $147 \mathrm{p}$.

FERNANDES, A. A. Chuva de sementes em trechos de diferentes estádios sucessionais da Mata Atlântica no município de Miguel PereiraRJ. 2006. 31 f. Monografia (Engenharia Florestal) - Universidade Federal Rural do Rio de Janeiro, 
Seropédica, 2006.

GOMES, J. F. et al. Classificação e crescimento de unidades de vegetação em Floresta Ombrófila Mista, São Francisco de Paula, RS. Ciência Florestal, Santa Maria, v. 18, n. 1, p. 93-107, jan./mar. 2008. HARPER, J. L. Population biology of plants. London: Academic Press, 1977. 892 p.

KAGEYAMA, P. Y.; PIÑA-RODRIGUES, F. C. M. Fatores que afetam a produção de sementes. In: AGUIAR, I. B.; PIÑA-RODRIGUES, F. C. M.; FIGLIOLIO, M. D. (Ed.). Sementes tropicais florestais. Brasília: ABRATES, 1993. p. 19-46.

JACKSON, J. F. Seasonality of flowering and leaf fall in a Brazilian subtropical lower montane moist forest. Biotropica, Oxford, v. 10, p. 38-42, 1978.

LARCHER, W. Ecofisiologia vegetal. São Carlos: RIMA, 2000. $531 \mathrm{p}$.

LEITE, P. F.; KLEIN, R. M. Vegetação. In: IBGE. Geografia do Brasil: Região Sul. Rio de Janeiro: IBGE, 1990. p. 113-150.

LEMOS, R. C. et al. Levantamento e reconhecimento dos solos do Estado do Rio Grande do Sul. Recife: Ministério da Agricultura, Departamento Nacional de Pesquisas Agropecuárias - Divisão de Pesquisas Pedológicas, 1973. 431 p. (Boletim Técnico, 30).

LONGHI, S. J. et al. Aspectos fitossociológicos de fragmento de Floresta Estacional Decidual, Santa Maria, RS. Ciência Florestal, Santa Maria, v. 10, n. 2, p. 59-74, 2000.

LONGHI, S. J. et al. Classificação e caracterização de estágios sucessionais em remanescentes de Floresta Ombrófila Mista na flona de São Francisco De Paula, RS, Brasil. Ciência Florestal, Santa Maria, v. 16, n. 2, p. 113-125, 2006.

LORENZI, H. Árvores brasileiras: manual de identificação e cultivo de plantas arbóreas nativas do Brasil. Nova Odessa: Instituto Plantarum, 2002a. v. $1,381 \mathrm{p}$.

LORENZI, H. Árvores brasileiras: manual de identificação e cultivo de plantas arbóreas nativas do Brasil. Nova Odessa: Instituto Plantarum, 2002b. v. 2, $381 \mathrm{p}$.

MARTÍNEZ-RAMOS, M.; SOTO-CASTRO, A. Seed rain and advanced regeneration in a tropical rain forest. Vegetation, Berlin, v. 107/108, p. 299-318, 1993.

PIVELLO, V. R. et al. Chuva de sementes em fragmentos de Floresta Atlântica (São Paulo, SP, Brasil), sob diferentes situações de conectividade, estrutura florestal e proximidade da borda. Acta Botanica Brasilica, São Paulo, v. 20, n. 4, p. 845-859, 2006.

PUIG, H. Floresta tropical úmida: São Paulo: UNESP, 2008. 496 p.

SCCOTI, M. S. V. et al. Dinâmica da vegetação em remanescente de Floresta Estacional Subtropical. 2012. 177 f. Tese (Doutorado em Engenharia Florestal) - Universidade Federal de Santa Maria, Santa Maria, 2012.

SCHERER, C. J. A.; JARENKOW, J. A. Banco de sementes em floresta estacional no Rio Grande do Sul. Revista Brasileira de Botânica, São Paulo, v. 29, n. 1, p. 67-77, jan./mar. 2006.

SCHUMACHER, M. V. et al. (Eds.) A Floresta Estacional Subtropical - Caracterização e Ecologia no Rebordo do Planalto Meridional. Santa Maria: Pallotti, 2011. 320 p.

SIMPSON, R. L. et al. Seed banks: general concepts and a methodological issues. In: LECK, M. A.; PARKER, T. V.; SIMPSON, R. L. (Ed.). Ecology of soil seed banks. New York: Academic Press, 1989. p. 3-8.

STRECK, E. D. et al. Solos do Rio Grande do Sul. Porto Alegre: EMATER/RSASCAR, 2008. 222 p.

VACCARO, S. et al. Aspectos da composição florística e categorias sucessionais do estrato arbóreo de três Subseres de uma Floresta Estacional Decidual, no Município de Santa Tereza - RS. Ciência Florestal, Santa Maria, v. 9, n. 1, p. 1-18, 1999.

VAN DER PIJL, L. Principles of dispersal in higher plants. Berlin: Springer Verlag, 1982. 162 p. VELOSO, H. P. et al. Classificação da vegetação brasileira, adaptada a um sistema universal. Rio de Janeiro: IBGE, 1991. 124 p.

VIEIRA, I. C. G. Dinâmica de sementes e regeneração vegetative em Floresta Sucessionais da Amazônia Oriental. In: GUARIGUATA, M. R.; FINEGAN, B. (Eds.). Ecology and management of tropical secondary: science, people, and policy. Proccedings of a conference held at Catie. Costa Rica: Turrialba, 1998. 244 p. 